\title{
BANACH SPACES THAT HAVE NORMAL STRUCTURE AND ARE ISOMORPHIC TO A HILBERT SPACE
}

\author{
JAVIER BERNAL AND FRANCIS SULLIVAN
}

\begin{abstract}
We prove that given a Hilbert space $(E,\|\cdot\|)$, and $|\cdot|$ a norm on $E$ such that for all $x \in E, 1 / \beta|x| \leqslant\|x\| \leqslant|x|$ for some $\beta$, if $1 \leqslant \beta<\sqrt{2}$, then $(E,|\cdot|)$ satisfies a convexity property from which normal structure follows.
\end{abstract}

1. Introduction. A Banach space $E$ is said to have normal structure if for each bounded, closed and convex subset $C$ of $E$, consisting of more than one point, there is an $x \in C$ such that

$$
\sup \{\|x-y\|: y \in C\}<\operatorname{diam}(C) \equiv \sup \left\{\left\|y_{1}-y_{2}\right\|: y_{1}, y_{2} \in C\right\} .
$$

In [4] it was proved that if $E$ has normal structure, $C \subseteq E$ is a nonempty weakly compact convex set, and $T: C \rightarrow C$ is a mapping such that for all $x, y \in C$, $\|T x-T y\| \leqslant\|x-y\|$, then $T$ has a fixed point in $C$.

For $r \geqslant 1$ let $E_{r}$ be the space $l_{2}$ renormed by

$$
|x|_{r}=\max \left\{\|x\|_{2}, r\|x\|_{\infty}\right\} \text {, }
$$

where $\|\cdot\|_{2}$ and $\|\cdot\|_{\infty}$ denote the $l_{2}$ and $l_{\infty}$ norms, respectively. It is known from [1] that $E_{r}$ has normal structure when $r<\sqrt{2}$.

We use the idea of multidimensional volumes and their related convexity moduli to prove $E_{r}$ satisfies a convexity property that implies this result. The notion of volumes in Banach spaces was introduced by Silverman and its use in defining moduli of convexity was introduced in [5]. Roughly speaking, the modulus of $k$-rotundity, $\delta_{k}(\varepsilon)$, measures the depth below the surface of the unit sphere of the centroid of a simplex of $k+1$ norm-1 vectors enclosing a $k$-dimensional volume larger than $\varepsilon$. In symbols,

$$
A\left(x_{1}, \ldots, x_{k+1}\right) \geqslant \varepsilon
$$

implies that

$$
\left\|\left(x_{1}+\cdots+x_{k+1}\right) /(k+1)\right\| \leqslant 1-\delta_{k}(\varepsilon) .
$$

Here $A\left(x_{1}, \ldots, x_{k+1}\right)$ denotes the enclosed volume. In case $k=1, A\left(x_{1}, x_{2}\right)=$ $\left\|x_{1}-x_{2}\right\|$ and $\delta_{1}(\varepsilon)$ is the usual modulus of convexity. In all cases

$$
D\left(\|\cdot\|, x_{1}, \ldots, x_{k+1}\right) \leqslant A\left(x_{1}, \ldots, x_{k+1}\right) .
$$

Received by the editors May 25, 1983

1980 Mathematics Subject Classification. Primary 46B20; Secondary 46C05. 
Here

$$
\begin{aligned}
D\left(\|\cdot\|, x_{1}, \ldots, x_{k+1}\right) \equiv & \left\|x_{k}-x_{k+1}\right\| \cdot \operatorname{dist}\left(x_{k-1},\left[x_{k}, x_{k+1}\right]\right) \\
& \ldots \cdot \operatorname{dist}\left(x_{1},\left[x_{2}, \ldots, x_{k+1}\right]\right)
\end{aligned}
$$

where $\left[x_{i+1}, \ldots, x_{k+1}\right]$ is the affine span of the vectors $x_{i+1}, \ldots, x_{k+1}$ and

$$
\operatorname{dist}\left(x_{i},\left[x_{i+1}, \ldots, x_{k+1}\right]\right)=\inf \left\{\left\|x_{i}-x\right\|: x \in\left[x_{i+1}, \ldots, x_{k+1}\right]\right\} .
$$

For a Hilbert space the inequality is always equality.

A connection between these moduli and normal structure of a Banach space $E$ was given in [3], namely

LemMA. Suppose that for some $\delta>0$ and some $0<\varepsilon<1$ there is an integer $m$ such that for all norm $-1 x_{1}, \ldots, x_{m} \in E$, if $\left\|\left(x_{1}+x_{2}+\cdots+x_{m}\right) / m\right\|>1-\delta$ then $D\left(\|\cdot\|, x_{1}, \ldots, x_{m}\right)<\varepsilon$.

Then $E$ is super-reflexive and has normal structure.

\section{The result.}

TheOrem. Let $(E,\|\cdot\|)$ be a Hilbert space, and let $|\cdot|$ be a norm on $E$ such that for all $x \in E, 1 / \beta|x| \leqslant\|x\| \leqslant|x|$ for some $\beta, 1 \leqslant \beta<\sqrt{2}$. Given $\varepsilon>0$, there exists $\delta>0$ and $M$, a positive integer, such that for $m \geqslant M$, if $x_{1}, \ldots, x_{m} \in E,\left|x_{1}\right|, \ldots,\left|x_{m}\right|$ $\leqslant 1$, and $\left|\left(x_{1}+\cdots+x_{m}\right) / m\right|^{2}>1-\delta$, then $D\left(|\cdot|, x_{1}, \ldots, x_{m}\right)<\varepsilon$.

The proof requires some preliminary results.

LEMMA 1. Given $k$, a positive integer and $\beta>0$, let $f, g$ be the functions from $R^{k}$ into $R$ defined by

$$
\begin{aligned}
& f\left(x_{1}, \ldots, x_{k}\right)=\frac{1}{k+1} \sum_{i=1}^{k} \frac{i}{i+1}\left(x_{k+1-i}\right)^{2}, \\
& g\left(x_{1}, \ldots, x_{k}\right)=\beta-\prod_{i=1}^{k} x_{i}, \quad\left(x_{1}, \ldots, x_{k}\right) \in R^{k} .
\end{aligned}
$$

Then $f(x) \geqslant(k /(k+1))\left(\beta^{2 / k} /(k+1)^{1 / k}\right)$ whenever $x \in R^{k}$ and $g(x)=0$.

Proof. Let $w \in \Omega \equiv\left\{x \in R^{k}: g(x)=0\right\}$ and $y=f(w)>0$. Then $\hat{\Omega} \equiv$ $f^{-1}([0, y]) \cap \Omega$ is nonempty and compact and, thus, there exists $x^{*} \in \hat{\Omega}$, a global minimum point of $f$ over $\hat{\Omega}$. Also, if $z \in \Omega \backslash f^{-1}([0, y])$ then $f(z)>y$ so that $x^{*}$ is a global minimum point of $f$ over $\Omega$.

With $x^{*}=\left(b_{1}, \ldots, b_{k}\right)$ then $\prod_{i=1}^{k} b_{i}=\beta>0$. Thus $\nabla g\left(x^{*}\right) \neq 0$, where $\nabla g$ is the gradient of $g$. It now follows, by Lagrange's theorem, that for some $\lambda \in R$,

$$
\nabla f\left(x^{*}\right)=\lambda \nabla g\left(x^{*}\right) .
$$

So, for each $i, 1 \leqslant i \leqslant k$,

$$
\frac{2}{k+1} \frac{k+1-i}{k+2-i} b_{i}=\lambda \prod_{\substack{j=1 \\ j \neq i}}^{k} b_{j}
$$


or

$$
\frac{2}{k+1} \frac{k+1-i}{k+2-i} b_{i}^{2}-\lambda \beta=0
$$

Thus, for each $i, 1 \leqslant i \leqslant k,((k+1) / 2) \lambda \beta=((k+1-i) /(k+2-i)) b_{i}^{2}$, and

$$
\left[\frac{k+1-i}{k+2-i} b_{i}^{2}\right]^{k}=\prod_{j=1}^{k} \frac{k+1-j}{k+2-j} b_{j}^{2}=\frac{1}{k+1} \beta^{2} .
$$

Therefore, $((k+1-i) /(k+2-i)) b_{i}^{2}=\beta^{2 / k} /(k+1)^{1 / k}$ for each $i, 1 \leqslant i \leqslant k$. So, given $x \in \Omega$,

$$
\begin{aligned}
f(x) & \geqslant f\left(x^{*}\right)=\frac{1}{k+1} \sum_{i=1}^{k} \frac{i}{i+1} b_{k+1-i}^{2} \\
& =\frac{1}{k+1} \sum_{i=1}^{k} \frac{k+1-i}{k+2-i} b_{i}^{2}=\frac{k}{k+1} \frac{\beta^{2 / k}}{(k+1)^{1 / k}} .
\end{aligned}
$$

Lemma 2. Let $(E,\|\cdot\|)$ be a Hilbert space and $k$ any positive integer. Given $x_{1}, \ldots, x_{k+1} \in E,\left\|x_{1}\right\|, \ldots,\left\|x_{k+1}\right\| \leqslant 1$, then

$$
\left\|\frac{x_{1}+\cdots+x_{k+1}}{k+1}\right\|^{2} \leqslant 1-\frac{1}{k+1} \sum_{i=1}^{k} \frac{i}{i+1}\left\|x_{k+1-i}-\frac{\sum_{j=k+2-i}^{k+1} x_{j}}{i}\right\|^{2} .
$$

Proof. Since $(E,\|\cdot\|)$ is a Hilbert space, given $x, y \in E$, then

$$
\left\|\frac{1}{k+1} x+\frac{k}{k+1} y\right\|^{2}=\frac{1}{k+1}\|x\|^{2}+\frac{k}{k+1}\|y\|^{2}-\frac{k}{(k+1)^{2}}\|x-y\|^{2} .
$$

In particular, given $x_{1}, \ldots, x_{k+1} \in E$,

$$
\begin{aligned}
\left\|\frac{x_{1}+\cdots+x_{k+1}}{k+1}\right\|^{2}= & \frac{1}{k+1}\left\|x_{1}\right\|^{2}+\frac{k}{k+1}\left\|\frac{x_{2}+\cdots+x_{k+1}}{k}\right\|^{2} \\
& -\frac{k}{(k+1)^{2}}\left\|x_{1}-\frac{x_{2}+\cdots+x_{k+1}}{k}\right\|^{2} .
\end{aligned}
$$

The proof of the lemma now follows by induction on $k$.

LEMMA 3. Let $(E,\|\cdot\|)$ be a Hilbert space and $k$ a positive integer. If $x_{1}, \ldots, x_{k+1} \in$ $E,\left\|x_{1}\right\|, \ldots,\left\|x_{k+1}\right\| \leqslant 1, D\left(\|\cdot\|, x_{1}, \ldots, x_{k+1}\right) \geqslant \varepsilon>0$, then

$$
\left\|\left(x_{1}+\cdots+x_{k+1}\right) /(k+1)\right\|^{2} \leqslant 1-(k /(k+1))\left(\varepsilon^{2 / k} /(k+1)^{1 / k}\right) .
$$

Proof. Since $D\left(\|\cdot\|, x_{1} \cdots x_{k+1}\right) \geqslant \varepsilon$ then $D\left(\|\cdot\|, x_{1}, \ldots, x_{k+1}\right)=\beta$, where $\beta \geqslant$ $\varepsilon$. Let $d_{i}=\operatorname{dist}\left(x_{i},\left[x_{i+1}, \ldots, x_{k+1}\right]\right)$, for each $i, 1 \leqslant i \leqslant k$. By Lemma 2 , with $f$ as defined in Lemma 1, it follows that

$$
\begin{aligned}
\left\|\frac{x_{1}+\cdots+x_{k+1}}{k+1}\right\|^{2} & \leqslant 1-\frac{1}{k+1} \sum_{i=1}^{k} \frac{i}{i+1}\left\|x_{k+1-i}-\frac{\sum_{j=k+2-i}^{k+1} x_{j}}{i}\right\|^{2} \\
& \leqslant 1-\frac{1}{k+1} \sum_{i=1}^{k} \frac{i}{i+1} d_{k+1-i}^{2}=1-f\left(d_{1}, \ldots, d_{k}\right) .
\end{aligned}
$$


However, $\prod_{i=1}^{k} d_{i}=D\left(\|\cdot\|, x_{1}, \ldots, x_{k+1}\right)=\beta$. So, by Lemma 1 ,

$$
f\left(d_{1}, \ldots, d_{k}\right) \geqslant(k /(k+1))\left(\beta^{2 / k} /(k+1)^{1 / k}\right),
$$

and, therefore,

$$
\left\|\left(x_{1}+\cdots+x_{k+1}\right) /(k+1)\right\|^{2} \leqslant 1-(k /(k+1))\left(\varepsilon^{2 / k} /(k+1)^{1 / k}\right) .
$$

REMARK. Extending these ideas [2] gives the exact value of the modulus of $k$-rotundity of a Hilbert space, e.g.

$$
\delta_{k}(\varepsilon)=1-\left[1-\frac{k}{k+1} \frac{\varepsilon^{2 / k}}{(k+1)^{1 / k}}\right]^{1 / 2} .
$$

Proof of The Theorem. Choose $\eta>0$ so that $\beta^{2}+\eta<2$. Given any $\varepsilon>0, k$ a positive integer, let $\Delta_{k}(\varepsilon)=(k /(k+1))\left(\varepsilon^{2 / k} /(k+1)^{1 / k}\right)$. Since $\lim _{k \rightarrow \infty} \Delta_{k}(\varepsilon)=1$, select $M>1$ so large that $\Delta_{m-1}(\varepsilon)>1-\eta$ whenever $m \geqslant M$.

Now, let $\delta=2-\beta^{2}-\eta$, and suppose $m \geqslant M,\left|\left(x_{1}+\cdots+x_{m}\right) / m\right|^{2}>1-\delta$, $\left|x_{1}\right|, \ldots,\left|x_{m}\right| \leqslant 1$, while $D\left(|\cdot|, x_{1}, \ldots, x_{m}\right) \geqslant \varepsilon$. Then $\left\|x_{1}\right\|, \ldots,\left\|x_{m}\right\| \leqslant 1$ and

$$
D\left(\|\cdot\|, x_{1}, \ldots, x_{m}\right) \geqslant(1 / \beta)^{m-1} \cdot D\left(|\cdot|, x_{1}, \ldots, x_{m}\right) \geqslant(1 / \beta)^{m-1} \varepsilon .
$$

It follows from Lemma 3 that

$$
\left\|\frac{x_{1}+\cdots+x_{m}}{m}\right\|^{2} \leqslant 1-\Delta_{m-1}\left(\left(\frac{1}{\beta}\right)^{m-1} \varepsilon\right) .
$$

However,

$$
\begin{aligned}
1-\delta & <\left|\frac{x_{1}+\cdots+x_{m}}{m}\right|^{2} \leqslant \beta^{2}\left\|\frac{x_{1}+\cdots+x_{m}}{m}\right\|^{2} \\
& \leqslant \beta^{2}\left(1-\Delta_{m-1}\left(\left(\frac{1}{\beta}\right)^{m-1} \varepsilon\right)\right)=\beta^{2}\left(1-\left(\frac{1}{\beta}\right)^{2} \Delta_{m-1}(\varepsilon)\right) \\
& =\beta^{2}-\Delta_{m-1}(\varepsilon)<\beta^{2}+\eta-1 .
\end{aligned}
$$

This contradicts the definition of $\delta$. Therefore, $D\left(|\cdot|, x_{1}, \ldots, x_{m}\right)<\varepsilon$. Q.E.D.

The result proven in [1] now follows from this theorem and the lemma mentioned in the introduction.

Corollary. Let $(E,\|\cdot\|)$ be a Hilbert space and let $|\cdot|$ be a norm on $E$ such that for all $x \in E, 1 / \beta|x| \leqslant\|x\| \leqslant|x|$ for some $\beta, 1 \leqslant \beta<\sqrt{2}$. Then $(E,|\cdot|)$ has normal structure.

Note that the Theorem is sharp because Baillon and Schöneberg proved that $E_{r}$ fails to have normal structure for $r \geqslant \sqrt{2}$. 


\section{REFERENCES}

1. J. B. Baillon and R. Schöneberg, Asymptotic normal structure and fixed points of nonexpansive mappings, Proc. Amer. Math. Soc. 81 (1981), 257-264.

2. J. Bernal, Behavior of $k$-dimensional convexity moduli, Thesis, Catholic University of America, Washington, D.C., 1980.

3. J. Bernal and F. Sullivan, Multi-dimensional volumes, super-reflexivity and normal structure in Banach space, Illinois J. Math. 27 (1983), 501-513.

4. W. A. Kirk, A fixed point theorem for mappings which do not increase distances, Amer. Math. Monthly 72 (1965), 1004-1006.

5. F. Sullivan, A generalization of uniformly rotund Banach spaces, Canad. J. Math. 31 (1979), 628-636.

Center for Applied Mathematics, National Bureau of Standards, Washington, D. C. 20234 